\title{
Micro OLED rapide sur électrodes coplanaires pour un fonctionnement en régime d'excitation impulsionnelle nanoseconde
}

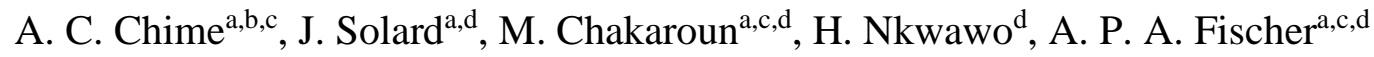 \\ ${ }^{a}$ Université Sorbonne Paris Cité, Université Paris 13, Laboratoire de Physique des \\ Lasers, UMR CNRS 7538, 99 avenue JB. Clément, 93430 Villetaneuse, France. \\ ${ }^{\mathrm{b}}$ Université de Dschang, IUT-FV, Bandjoun, Cameroun \\ ${ }^{\mathrm{c}}$ Labex SEAM (Science Engineering and Advanced Materials), 99 Avenue Jean \\ Baptiste Clément, 93430 Villetaneuse \\ ${ }^{\mathrm{d}}$ Université Sorbonne Paris Cité, Université Paris 13, Centrale de Proximité en \\ Nanotechnologies de Paris Nord, 99 avenue JB. Clément, 93430 Villetaneuse, France
}

Contact email : fischer@univ-paris13.fr

\begin{abstract}
Dans ce travail, nous nous intéressons à la conception, à la réalisation de micro-OLEDs ultra rapides et à leur caractérisation en régime d'excitation électrique impulsionnelle nanoseconde. Les réponses ultra-rapides de ces $\mu$-OLED sont obtenues par des électrodes spécifiques empruntées aux techniques micro-ondes. Cette combinaison de l'électronique organique et des techniques micro-ondes permet d'atteindre des valeurs record en termes de durée d'impulsion d'excitation ultra courte $(5 \mathrm{~ns})$ et de densité de courant ultra-intense $\left(4,7 \mathrm{kA} / \mathrm{cm}^{2}\right)$. Ces résultats ouvrent des perspectives vers la réalisation de la première diode laser organique.
\end{abstract}

\section{Introduction}

L'un des enjeux actuels de l'optoélectronique organique est la réalisation de la diode laser organique. Celle-ci n'a pas encore été démontrée pour des raisons multiples. On peut citer entre autres la faible mobilité des porteurs dans les semiconducteurs organiques qui limitent les densités de courant. En régime continu, les valeurs typiques de densité de courant maximales dans les OLEDs sont comprises dans la plage de $100 \mathrm{~mA}$ à $1 \mathrm{~A} / \mathrm{cm}^{2}$ alors que les niveaux équivalents au seuil laser se situent bien au-delà de $1 \mathrm{kA} / \mathrm{cm}^{2}$. Pour atteindre ces niveaux d'excitation, et gagner trois ordres de grandeurs, travailler en régime impulsionnel est indispensable $[1,2]$.

La première contrainte sur la durée de l'impulsion concerne la destruction du composant par effet thermique. La deuxième contrainte trouve son origine dans les propriétés physiques du matériau organique électroluminescent ; au-delà de la dizaine de nanosecondes au sein des niveaux excités des matériaux organiques électroluminescents, est provoquée une accumulation des états triplets qui engendre de nouvelles pertes optiques. Ceci a pour effet de rehausser encore le seuil laser et par conséquent la densité d'excitation nécessaire pour l'atteindre.

L'objectif de ce travail est d'atteindre des densités de courant supérieur au $\mathrm{kA} / \mathrm{cm}^{2}$ dans des OLEDs soumises à des impulsions électriques de durée inférieure à la dizaine de nanoseconde. A cet ordre de grandeur, il faut prendre en compte les problématiques des hautes fréquences, notamment en termes d'adaptation d'impédance des lignes d'excitation et de bande passante du composant.

Nous présentons ici, la conception, la fabrication et la caractérisation d'un composant optoélectronique organique ultra-rapide incorporant des électrodes coplanaires spécifiquement adaptées sont ajoutées. L'originalité consiste ici à combiner électronique organique et électronique hyperfréquence. 


\section{Description de l'OLED}

L'OLED rapide est composée d'une anode structurée en ligne coplanaire (§2 .1) intégrant une résistance intégrée planaire (\$2.2) sur laquelle est déposée une hétérostructure organique $(\$ 2.3)$ laquelle est recouverte d'une cathode d'aluminium. Le composant est conçu avec une résistance planaire intégrée.

\section{$\underline{\text { Structuration de l'anode en ligne coplanaire CPW }}$}

Pour minimiser les réflexions des impulsions électrique, la géométrie de lignes coplanaires (Coplanar Waveguide - CPW), s'est imposée comme la solution la mieux adaptée aux substrats OLED faits de verre recouvert d'une électrode transparent et conductrice en raison de l'existence d'une seule face conductrice [3]. Une des nouveautés consiste ici à concevoir et réaliser ces lignes CPW dans un matériau transparent et conducteur ; un alliage d'oxyde d'étain et d'indium (ITO).

La figure 1 présente le principe d'une ligne CPW. Elle est constituée d'une bande conductrice centrale (Signal $-S$ ) placée à équidistance entre deux plans de masses (Ground - $G$ ), toutes disposées dans le même plan au-dessus d'un support diélectrique. $\boldsymbol{H}$ et $\boldsymbol{\epsilon}$ représentent respectivement l'épaisseur et la permittivité diélectrique relative du diélectrique (substrat de verre). $\boldsymbol{W}$ et $\boldsymbol{T}$ représentent respectivement la largeur et l'épaisseur de la bande centrale. $\boldsymbol{G}$ est la largeur du gap séparant la bande centrale des plans de masse. L'impédance caractéristique $\boldsymbol{Z}_{\boldsymbol{C}}$ d'une telle ligne dépend des paramètres $\boldsymbol{\epsilon}_{\boldsymbol{r}}, \boldsymbol{H}, \boldsymbol{W}$, et $\boldsymbol{G}$. Les détails de calcul peuvent être consultés dans les références [4-8].

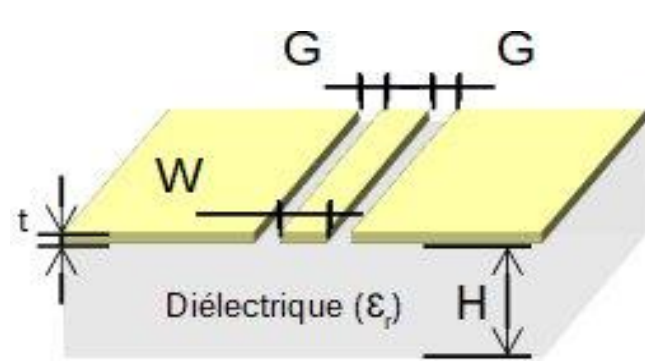

Fig.1. Schéma de principe d'une ligne coplanaire.

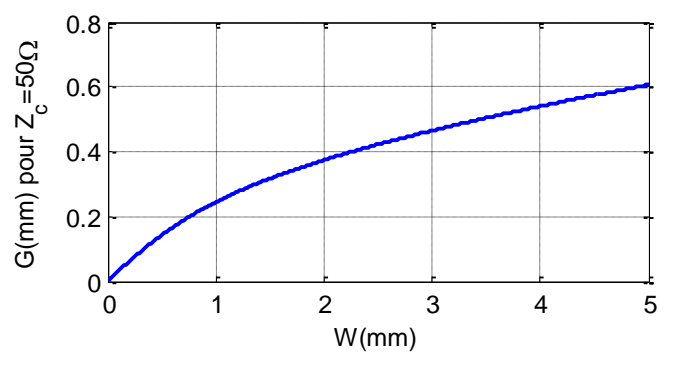

Fig.2. G(W) d'une ligne CPW d'impédance caractéristique $50 \Omega$.

La condition de continuité d'impédance caractéristique entre les électrodes et les câbles d'alimentation se traduit par $\boldsymbol{Z}_{\boldsymbol{C}}=50 \Omega$. Pour satisfaire cette condition, la valeur du gap $\boldsymbol{G}$ est donnée en fonction de $\boldsymbol{W}$ sur la courbe de la figure 3, pour un substrat de verre de permittivité relative 7,75 et d'épaisseur $0,7 \mathrm{~mm}$. En pratique, le choix d'un couple de valeurs $(\mathrm{G}=243 \mu \mathrm{m}, \mathrm{W}=1000 \mu \mathrm{m})$ est imposé par les dispositifs de caractérisation, et en l'occurrence par l'écartement des sondes d'excitation et de mesure qui limitent la séparation $\mathrm{G}-\mathrm{W}-\mathrm{G}$ à un maximum de $4 \mathrm{~mm}$.

\section{Intégration d'une résistance de mesure}

Afin d'éviter des réflexions parasites, une résistance $\boldsymbol{R}_{\boldsymbol{m}}$ dédiée à la mesure du courant est également intégrée au dispositif sous la forme de deux petites pistes reliant la ligne centrale et les deux plans de masses. Leurs largeurs $\boldsymbol{L}$ et longueurs $\boldsymbol{G}$ permettent de calculer la résistance de mesure,

$$
R_{m}=\frac{1}{2} R_{\text {ITO }} \frac{G}{L}
$$

où $\boldsymbol{R}_{\text {ITo }}$ représente la résistance par carré de l'ITO. 


\section{$\underline{\text { La structure de l'OLED }}$}

L'hétéro-structure qui constitue l'OLED est un empilement de composés organiques de 140nm d'épaisseur déposés sur l'anode CPW d'ITO et recouverts par une cathode d'aluminium. Les couches organiques successives jouent respectivement les rôles d'injecteur de trous (HIL), de transporteur de trous (HTL), d'émetteur (EML), de bloqueur de trous (HBL), et de transporteur d'électrons (ETL). Les matériaux sont choisis en fonction de leurs niveaux d'énergies HOMO (Highest Occupied Molecular Orbital) et LUMO (Lowest Unoccupied Molecular Orbital) et des mobilités des porteurs. Le diagramme d'énergie de l'hétéro-structure considérée peut être consulté dans la référence [9]. L'hétéro-structure utilisée dans ces travaux est constituée d'une couche de 30nm de m-MTDATA (4,4',4"-Tris[(3-methylphenyl) phenylamino] triphenylamine), d'une couche de 10nm de NPB (N,N'-Di(1-naphthyl)-N,N'-diphenyl(1,1'-biphenyl)-4,4'-diamine), et d'une couche de $100 \mathrm{~nm}$, sur lesquelles sont coévaporés de l'Alq3 (Tris-(8-hydroxyquinoline aluminum) et du DCM (4(Dicyanomethylene)-2-methyl-6-(4-dimethyl aminostyryl)-4H-pyran) avec une concentration de $1,5 \%$ en masse. Elle est terminée par $1 \mathrm{~nm}$ de fluorure de lithium (Lif) suivi de 100nm d'aluminium constituant la cathode. La surface active, de forme rectangulaire, est délimitée par la section de croisement entre les deux électrodes.

Plusieurs surfaces actives $\mathrm{S}=e . D$ existent $100 \times 150 \mu \mathrm{m}^{2}, 50 \times 150 \mu \mathrm{m}^{2}$ et $25 \times 150 \mu \mathrm{m}^{2}$.

La structure d'ensemble vue de dessus est présentée sur la figure 3 ; l'anode (A) et la cathode $(\mathrm{K})$ correspondent aux lignes centrales séparées des plans de masse (GND) par le gap $G$. L'empilement des couches organiques est représenté par un rectangle vert $(\mathrm{O})$, et la surface active ( $\underline{\mathrm{S}})$ de l'OLED représentée par des hachures a pour dimensions la largeur $D$ de la partie aluminium de la cathode et la largeur $e$ de l'extrémité de l'anode. La double résistance de mesure indiquée par $(\mathrm{R})$ qui relie la cathode $(\mathrm{K})$ au plan de masse (GND) a pour largeur $L$ et

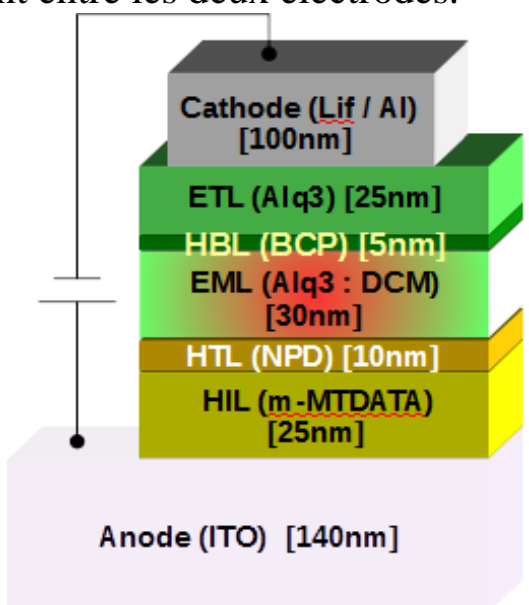

Fig.3. Structure d'une OLED longueur $G$ ce qui définit la résistance de mesure $R m$.

Dans l'expérience présentée ici, les valeurs précédemment choisies d'épaisseur de substrat $H=700 \mu \mathrm{m}$, d'épaisseur d'ITO $t=120 \mathrm{~nm}$, de largeur de piste centrale $W=1000 \mu \mathrm{m}$, et de gap $G=243 \mu \mathrm{m}$, conduisent à une largeur des résistances de $L=182 \mu \mathrm{m}$, pour une résistance de mesure $R_{m}=13 \Omega$. Davantage de détails sur la conception sont présentés dans la référence [9].

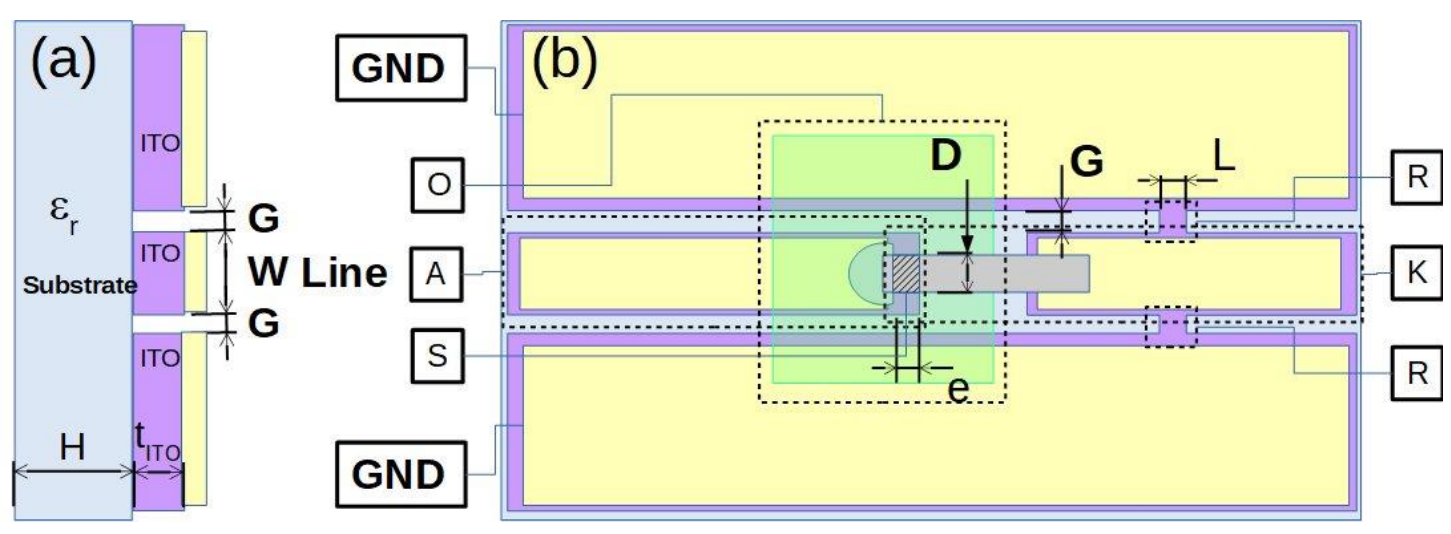

Fig.4. Structure d'une OLED ultra-rapide sur électrode coplanaire CPW. 


\section{III.Fabrication}

La fabrication des $\mu$-OLED se décompose en 3 phases principales. En phase 1, (ligne 1 de la figure 5), la face transparente et conductrice du substrat va subir des étapes d'enrésinement, de photolithographie suivies de structuration par gravure plasma pour définir la géométrie des électrodes intégrant la résistance de mesure. En phase 2, (ligne 2 de la figure 5), un lift-off par enrésinement, une photolithographie suivie du dépôt d'une double couche de chrome et d'or sur l'ITO structuré permet une métallisation locale sur les plans de masse et les lignes à l'exception de la zone active et des résistances de mesure. Ceci va permettre de minimiser les effets résistifs de l'ITO. La phase 3, est le dépôt par évaporation thermique des couches organiques et de la cathode d'aluminium décrite dans le paragraphe II.

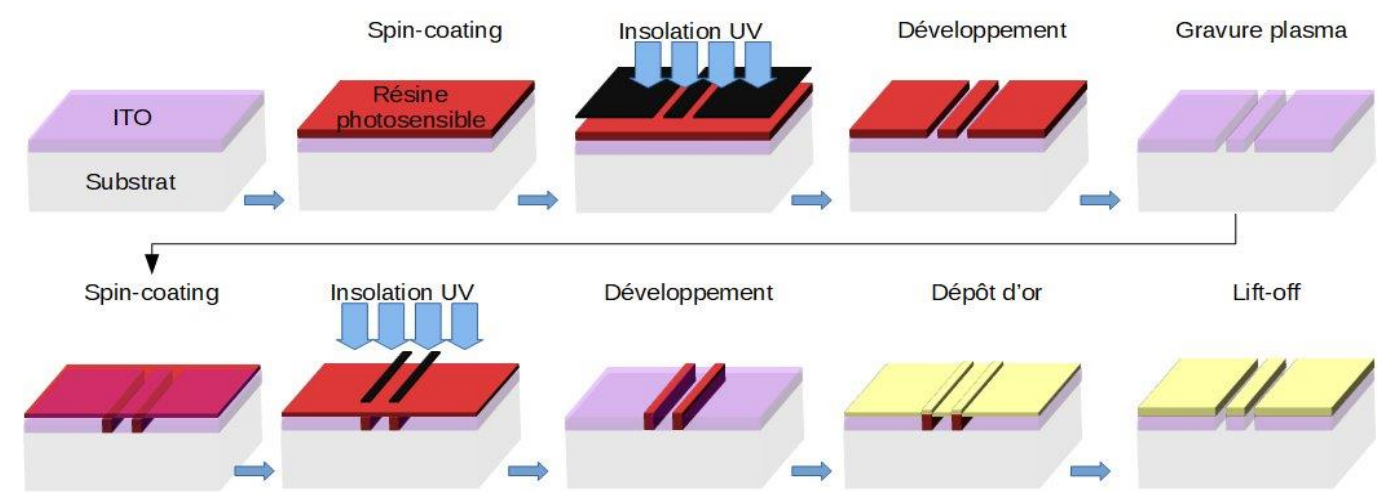

Fig.5. Etapes du procédé de fabrication de l'OLED sur électrode CPW.

Ce processus de fabrication repose essentiellement sur les procédés technologiques habituels de la microélectronique que sont la lithographie, la gravure, la métallisation locale par lift-off, et le dépôt par évaporation thermique de couches minces, mais ils sont appliqués pour la première fois à un composant résultant d'une combinaison d'optoélectronique organique et de techniques micro-ondes. Davantage de précision sur la fabrication sont présentées dans la référence [9].

\section{Résultats expérimentaux}

Trois types de caractérisations du dispositif sont réalisés permettant de valider les propriétés de la ligne $\mathrm{CPW}$, de mesurer la valeur de la résistance de mesure, d'observer les réponses électriques et optiques de l'OLED rapide, et de tracer la caractéristique courant-tension.

Tout d'abord les propriétés hyperfréquences des lignes et du dispositif sont mesurées avec un analyseur de réseau vectoriel (VNA). On caractérise pour cela une ligne CPW simple sans OLED terminée sur une charge résistive de $50 \Omega$. Cette ligne simple ne comporte pas les couches organiques mais présente une largeur $\mathrm{W}$ et un gap $\mathrm{G}$ identiques à ceux de l'OLED. La mesure en transmission effectuée à l'autre extrémité de la ligne est représentée sur la figure 6. Le nuage de points de l'abaque de Smith centré sur $(1,0)$ confirme un coefficient de réflexion (ou paramètre $\mathrm{S} 11$ ) très faible, et valide une impédance caractéristique de $Z c=50 \Omega$.

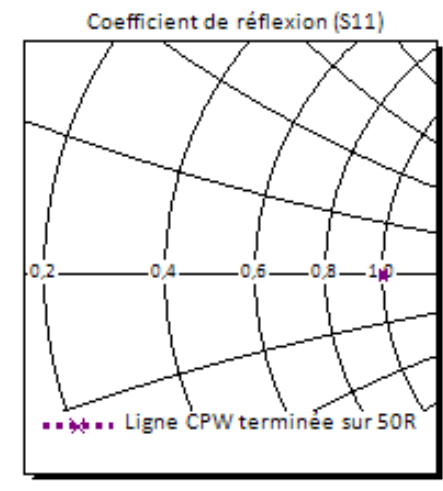

Fig.6. Coefficient de réflexion S11 de la ligne CPW. 
La résistance de mesure $R m=13 \Omega$ est mesurée tout d'abord en régime continu avec un simple voltmètre, puis dans un second temps avec un analyseur de réseau vectoriel en configuration de paramètre $\mathrm{S} 11$ à un seul port sur le même échantillon sans hétéro-structure organique. La valeur de l'impédance mesurée sur la plage de fréquence $30 \mathrm{kHz}$ à $3 \mathrm{GHz}$ se situe dans la plage $13,5 \Omega 13,9 \Omega-\mathrm{j} 1,5 \Omega$ en relativement bon accord avec la mesure en régime continu. Des mesures complémentaires permettent de mesurer la capacité de jonction de l'OLED, $\mathrm{C}=4,5 \mathrm{pF}$. L'étape de caractérisation suivante consiste à soumettre des $\mu$-OLEDs CPW comportant l'hétéro-structure organique définie au paragraphe II qui sont soumises à des impulsions électriques de durée $5 \mathrm{~ns}$ et d'amplitude variant entre $5 \mathrm{~V}$ et $50 \mathrm{~V}$ issues d'un générateur d'impulsion AVTECH AVL-2A-B; les réponses électriques et optiques sont observées avec un oscilloscope rapide Tektronix TDS 7254 et une photodiode à avalanche Thorlabs APD430A2 de 400MHz de bande passante.
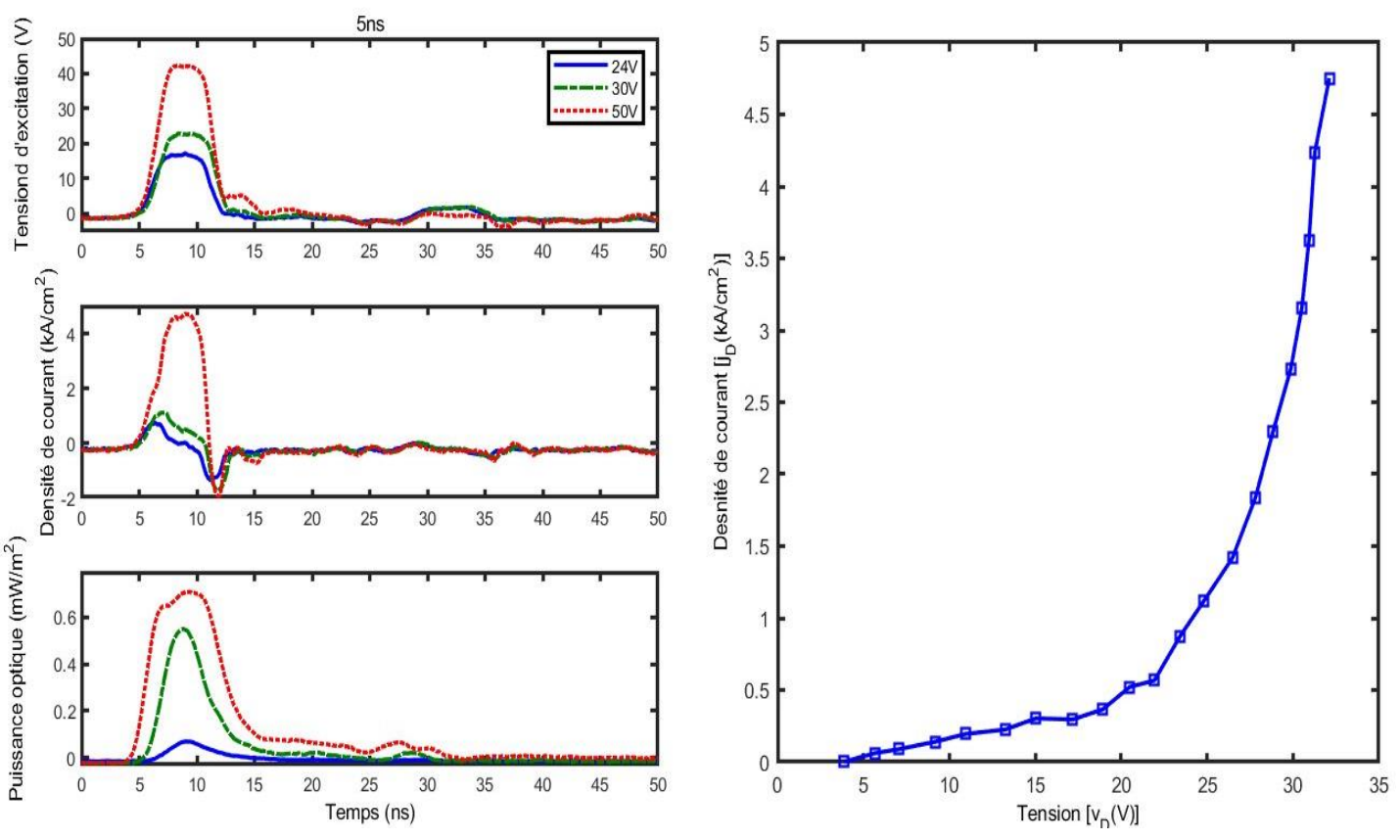

Fig.7. (a) Tension d'excitation, (b) réponses optiques et réponses électriques en densité de courant à des impulsions d'amplitude $24 \mathrm{~V}, 30 \mathrm{~V}$ et $50 \mathrm{~V}$; (b) Caractéristique $\mathrm{JD}(\mathrm{VD})$ construite à partir des mesures obtenues en régime d'impulsions.

La figure 7.a présente les mesures résolues temporellement de la tension d'excitation, de la réponse électrique en densité de courant et de la réponse optique pour un échantillon de taille $100 \times 150 \mu \mathrm{m}^{2}$ soumis à des impulsions de durée $5 \mathrm{~ns}$ et des amplitudes de $24 \mathrm{~V}, 30 \mathrm{~V}$ et $50 \mathrm{~V}$. Ce résultat constitue une première en termes de durée d'impulsion. La figure 7.b présente la densité de courant $J_{D}$ traversant l'hétérostructure organique en fonction de la tension $V_{D}$ aux bornes de l'OLED. La tension $V_{D}$ réellement appliquée aux bornes de l'OLED est calculée en tenant compte de la chute de tension aux bornes de la résistance de mesure $R_{m}$, tandis que le courant traversant réellement l'OLED est calculé en retranchant le courant de charge au courant de décharge de la capacité parasite de jonction C. Le maximum de densité de courant, $\mathrm{J}_{\text {Dmax }}=4,75 \mathrm{kA} / \mathrm{cm}^{2}$, est obtenu pour une tension aux bornes de l'OLED de 32V. Cette valeur est supérieure à la précédente mesure de densité de courant, $\mathrm{J}=3,6 \mathrm{kA} / \mathrm{cm}^{2}$, réalisée dans une OLED sans électrode CPW et qui constitue un nouveau record de densité de courant dans une diode électroluminescente organique [10]. 


\section{Conclusion}

Nous avons combiné pour la première fois les techniques de l'électronique microonde et de l'électronique organique dans la fabrication de micro-OLED, ce qui nous a permis de dépasser plusieurs records, notamment en termes de durée d'impulsion (5ns) et de densité de courant maximale $\left(J_{D \max }=4,75 \mathrm{kA} / \mathrm{cm} 2\right)$ supportée dans une OLED rapide. Le record de $J_{D \max }=4,75 \mathrm{kA} / \mathrm{cm}^{2}$ atteint dans le cadre de ce travail montre qu'il est possible de lever le verrou de la limitation de courant dans une hétéro-structure organique et d'envisager la démonstration de la diode laser organique. Parmi les perspectives envisagées, nous envisageons l'ajout à cette structure d'une cavité laser de type DFB qui offre à la fois une compatibilité avec la structure planaire du composant et également un facteur de qualité parmi les plus élevés possibles.

\section{Remerciements}

Remerciement à la Centrale de Proximité en Nanotechnologie de Paris Nord $\mathrm{C}(\mathrm{PN}) 2$ qui a servi d'environnement technologique à la réalisation de ce travail. Remerciement également à Dejan Kocic pour son soutien technique.

Ce travail a bénéficié d'une aide publique par l'Agence Nationale de la Recherche dans le cadre du programme d'Investissement d'Avenir (Reference ANR-11-IDEX0005-02) via le Labex SEAM (Science Engineering and Advanced Materials).

\section{Références}

1. D. Kasemann, R. Brückner, H. Fröb, and K. Leo, "Organic light-emitting diodes under high currents explored by transient electroluminescence on the nanosecond scale," Physical Review $B, 84,11,(2011)$.

2. I. D. W. Samuel and G. A. Turnbull, "Organic Semiconductor Lasers," Chemical Reviews, 107(4), 1272 (2007).

3. C. P. Wen, "Coplanar waveguide: A surface strip transmission line suitable for nonreciprocal gyromagnetic device applications," IEEE Transactions on Microwave Theory and Techniques, ,17(12), 1087, (1969).

4. R. Simons, Coplanar waveguide circuits, components, and systems. New York : John Wiley, (2001).

5. S. S. Bedair and I. Wolff, "Fast, accurate and simple approximate analytic formulas for calculating the parameters of supported coplanar waveguides for (M) MIC's," IEEE Transactions on Microwave Theory and Techniques, 40(1), 41, (1992).

6. G. Ghione and C. U. Naldi, "Coplanar waveguides for MMIC applications: Effect of upper shielding, conductor backing, finite-extent ground planes, and line-to-line coupling," IEEE transactions on Microwave Theory and Techniques, 35(3), 260, (1987).

7. G. Ghione and C. Naldi, "Analytical formulas for coplanar lines in hybrid and monolithic MICs," Electronics Letters, 20(4), 179, (1984).

8. W. Hilberg, "From approximations to exact relations for characteristic impedances," IEEE Transactions on Microwave Theory and techniques, 17(5), 259 (1969).

9. A. C. Chime, S. Bensmida, M. Chakaroun, M. W. Lee, H. Nkwawo, and A. P. A. Fischer, "Electrical modelling and design of ultra-fast micro-OLED with coplanar wave-guided electrodes in ON-OFF regime", Organic Eletronic, 56, 284 (2018).

10. L. Zeng, A. C. Chime, M. Chakaroun, S. Bensmida, H. Nkwawo, A. Boudrioua, and A. P. A. Fischer,"Electrical and Optical Impulse Response of High-Speed Micro-OLEDs Under UltraShort Pulse Excitation “, IEEE Trans. Electron Devices, 64(7), 2942 (2017). 